\title{
Angimiolipoma renal como causa de shock hemorrágico agudo
}

\author{
López Fernández A, Lacasta García JD, Puig Giro R, Vicente de Vera P. \\ Servicio de Urgencias. Hospital Universitario Arnau de Vilanova. Lérida.
}

Actas Urol Esp 2008;32(8):855-857

\section{RESUMEN}

\section{ANGIMIOLIPOMA RENAL COMO CAUSA DE SHOCK HEMORRÁGICO AGUDO}

Presentamos una caso de hematoma retroperitoneal y shock hipovolémico como forma de presentación de angiomiolipoma renal, tumor benigno poco frecuente y generalmente asintomático. El sangrado masivo y espontáneo del tumor, fue diagnosticado mediante la realización de ecografía abdominal y confirmado por TAC abdominal de urgencia. Pese a que el tratamiento de este tipo de tumores, en general, es conservador, el tamaño y la severidad de la clínica acompañante obligó a trasladar a la paciente, una vez estabilizada hemodinámicamente, a hospital de tercer nivel para realizar embolización selectiva de la masa renal. La evolución fue favorable sin necesidad de realizar maniobras quirúrgicas más agresivas ni extirpación del angiomiolipoma de forma urgente.

Palabras clave: Angiomiolipoma. Shock. Riñon. Tumor.

\section{ABSTRACT}

\section{KIDNEY ANGIOMYOLIPOMA AS AETIOLOGY OF HAEMORRAGIC SHOCK}

This is a case of retroperitoneal hematoma and hypovolemic shock as a presentation of renal angiomyolipoma, a rarely benign and asymptomatic tumor.

The massive and spontaneous bleeding of the tumor was diagnosed by an abdominal ecography and confirmed by an abdominal computer tomography in the emergency room.

Although that kind of tumor needs non-aggressive treatment, as a general rule, because of the size and severity of the following symptoms, the patient has been submitted to a selective embolization of the renal mass when he was hemodinamically stabilized.

The evolution was favorable; it was not necessary to do more aggressive surgical handling neither to urgently remove the angiomyolipoma.

Keyword: Angiomyolipoma. Shock. Kidney. Tumor.

$\mathrm{L}^{-1}$ os angiomiolipomas son tumores benignos renales, generalmente son asintomáticos y diagnosticados de forma casual. En escasas ocasiones debutan con un cuadro agudo que compromete la vida del paciente, debido a sangrado espontáneo intratumoral.

Presentamos un caso de angiomiolipoma renal gigante que originó shock hemorrágico masivo intraabdominal.

\section{CASo CLÍnico}

Mujer de 71 años de edad sin antecedentes patológicos de interés, que ingresa en la sala de Reanimación del Servicio de Urgencias del Hospital Uni- versitario Arnau de Vilanova de Lleida, por dolor abdominal agudo, sensación de mareo, frialdad cutánea, diaforesis y signos de mala perfusión tisular con hipotensión de 70/40 mmHg y taquicardia a 120 pulsaciones por minuto. La exploración revelaba un abdomen blando y depresible con una gran masa en hipocondrio derecho no pulsátil. En la analítica destacaba hemoglobina de 9,5 hematocrito de $28,8 \%$, urea de $59 \mathrm{mg} / \mathrm{dl}$, creatinina de 1,47 y leucocitosis de 21200/1. con desviación a la izquierda. Las pruebas de coagulación resultaron normales. Se realiza Ecografía abdominal de urgencia que objetiva gran hematoma retroperitoneal derecho 
que depende del riñón a causa de angiomiolipoma. Tras estabiliza a la paciente mediante sobrecarga de volumen y concentrados de hematíes, se realiza TAC abdominal que confirmó el diagnóstico, apreciando la existencia de angiomiolipoma en polo inferior renal derecho (Fig. 1) con signos de sangrado intratumoral (Fig. 2) y hematomas en espacios perirrenales y pararrenales anterior y posterior con mínimo hemoperitoneo subhepático y en pelvis (Fig. 3). La paciente se deriva a hospital de tercer nivel, de urgencia, donde se le realizó embolización supraselectiva de la masa dependiente del riñón derecho. La paciente evolucionó favorablemente.

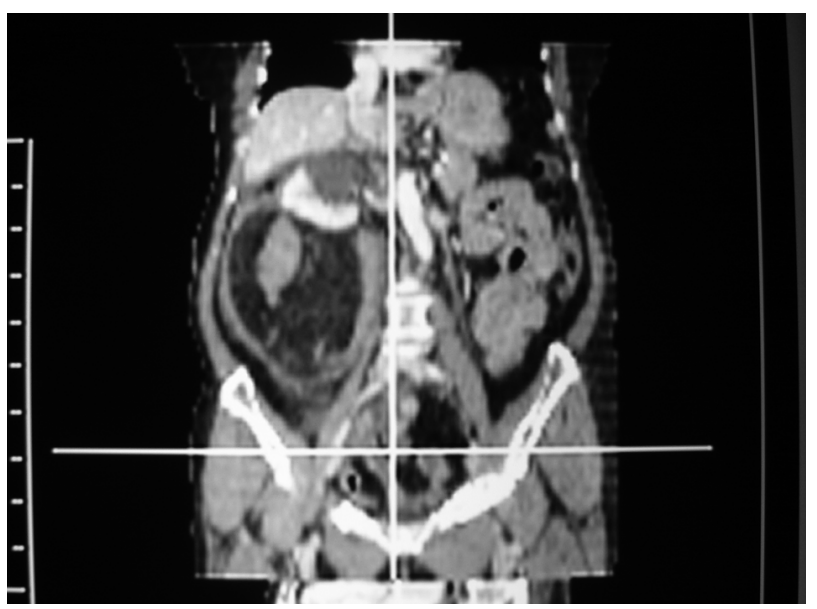

\section{FIGURA 1}

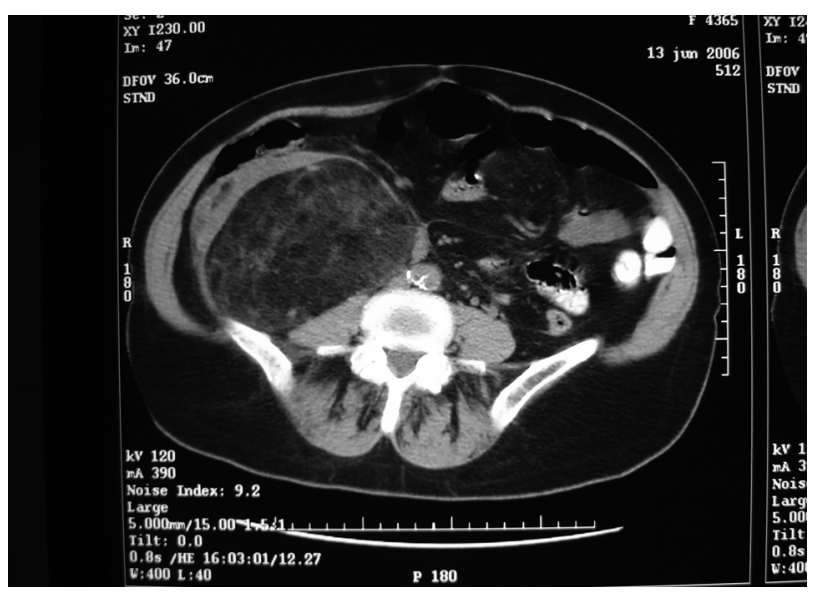

FIGURA 2

\section{DISCUSIÓN}

Los angiomiolipomas o hamartomas, son tumores benignos poco frecuentes a nivel renal, representando el 2-3\% de los tumores renales sólidos. La tercera parte de estos tumores presentan crecimiento extrarrenal, y el 15\% de los casos son bilaterales.

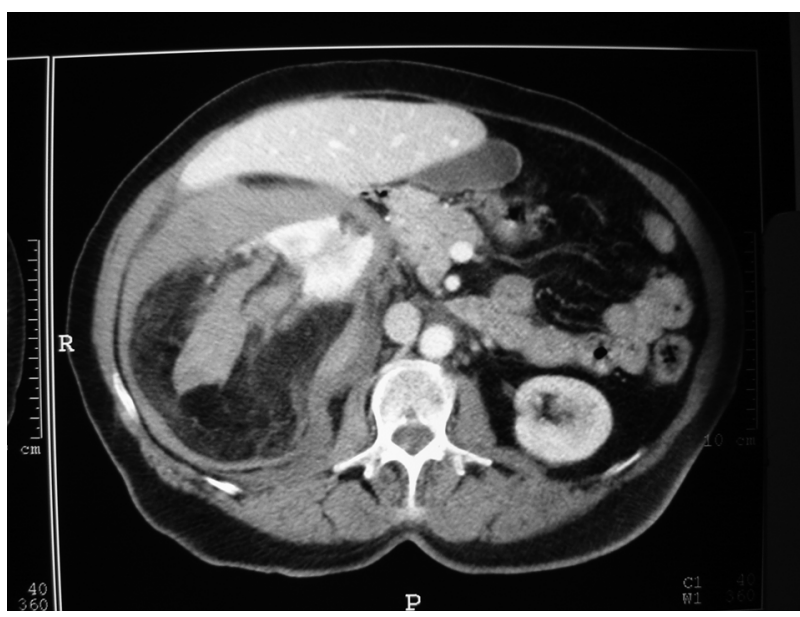

FIGURA 3

Están compuestos de tejido adiposo, vascular y músculo liso ${ }^{1}$.

Existen dos variantes de angiomiolipoma:

1. Aislado: $80 \%$ de los casos, más frecuente en mujeres (4:1) entre los 50 y los 70 años, y de presentación unilateral.

2. Asociado a facomatosis: $20 \%$ de los casos, como esclerosis tuberosa, enfermedad de Recklinghausen y a la de Von Piel Lindau, por orden de frecuencia, afectando en un 80\% al riñón y, el resto, a otras localizaciones, sin distinción entre sexos, y, a menudo, bilateral.

La clínica de estos tumores está relacionada, habitualmente, con el tamaño tumoral. Los de menos de $4 \mathrm{~cm}$ de diámetro, son asintomáticos ${ }^{3}$, hasta en un $60 \%$ de los casos. Cuando aparecen los sintomas, suelen ser tumores de más de $4 \mathrm{~cm}$ de diámetro, y consisten en dolor abdominal en flanco afecto, masa abdominal palpable y hematuria. En las formas más graves, cursa con abdomen agudo, hemoperitoneo y shock hipovolémico y, en ocasiones, sangrado espontáneo intratumoral, como en nuestro caso.

El diagnóstico se realiza mediante ecografía o TAC abdominal. El diagnóstico diferencial debe realizarse con el resto de tumores renales, especialmente, con el carcinoma renal. La ecografía y el TAC abdominal realizan el diagnóstico en el 90\% de los casos, siendo las técnicas más rentables. Otras técnicas de imagen empleadas para el diagnóstico del angiomiolipoma, son, la urografía excretora, la arteriografía, en la que se aprecia la típica imagen en "piel de cebolla", que se origina por las formaciones aneurismáticas y la presencia de vasos sanguíneos 
finos periféricos ${ }^{4}$; y la Resonancia Magnética, que es útil sólo en los casos en los que el tumor tiene una alta densidad de tejido adiposo y emite una señas de alta densidad.

El pronóstico de la lesión es bueno, pese a que se han descrito casos aislados de malignización con metástasis a distancia e infiltración local ${ }^{5}$.

El tratamiento, en general, es conservador cuando son de pequeño tamaño y asintomáticos. Si son mayores de $4 \mathrm{~cm}$ o muy sintomáticos, se realiza enucleación, embolización selectiva o nefrectomía parcial. La nefrectomía total se reserva para los casos de hemorragia retroperitoneal aguda, existencia de células carcinomatosas o cuando el riñón entero ha sido desplazado por el tumor ${ }^{6}$.

\section{REFERENCIAS}

1. Rosselló Barbará M, Mus Malleu A, Rebassa Llul M, Casals Armada J. Giant renal angiomyolipoma disclosed by mild lumbar trauma and treated with nephrectomy: report of a case. Actas Urol Esp. 2004,28(2):133-137.
2. Ng KF, Chen TC. Infiltrating renal angiomyolipoma into ascending colon associated with hepatic involvement in a patient with tuberous sclerosis. J Urol. 2001;165(1):179.

3. De Luca S, Terrone C, Rossetti SR. Management of renal angyomiolipoma: report of 53 cases. BJU Int. 1999;83(3):215-218.

4. Oesterling JE, Fishman EK, Golgman SM, Marshall FF. The management of renal angiomyolipoma. J Urol. 1986;135(6):11211124.

5. Ligeman JE, Donohue JP, Donohue JP, Madura JA, Selke F. Angiomyolipoma: Emerging concepts in management. Urology. 1982;20(6):566-570.

6. Gutiérrez Fernández G, Mansilla Roselló A, Rubio Gil F, Martínez Domínguez AP, Villar del Moral J, Ferrón Orihuela A et al. Angiomyolipoma. Actas Urol Esp. 2003;27(6):462-464.

Correspondencia autor: A. López Fernández Servicio de Urgencias

Hospital Universitario Arnau de Vilanova.

Avda. Alcalde Rovira Roure, 80 - 25198 Lérida

Tel.: 973248100

E-mail autor: mafaldaurora@yahoo.es

Información artículo: Nota Clínica

Trabajo recibido: febrero 2007

Trabajo aceptado: abril 2007 